\title{
CONTRIBUIÇÃO DAS FONTES DE SEDIMENTOS EM UMA BACIA HIDROGRÁFICA AGRÍCOLA SOB PLANTIO DIRETO ${ }^{(1)}$
}

\author{
Tales Tiecher ${ }^{(2)}$, Jean Paolo Gomes Minella ${ }^{(3)}$, Pablo Miguel ${ }^{(4)}$, Jimmy Walter Rasche \\ Alvarez $^{(5)}$, André Pellegrini ${ }^{(2)}$, Viviane Capoane ${ }^{(6)}$, Lucas Henrique Ciotti $^{(7)}$, Gilmar Luiz \\ Schaefer ${ }^{(7)} \&$ Danilo Rheinheimer dos Santos ${ }^{(3)}$
}

\section{RESUMO}

O conhecimento das principais fontes difusas de produção de sedimento pode aumentar a eficiência de utilização dos recursos públicos, investidos em estratégias de gestão em bacias hidrográficas, que visem mitigar a transferência de sedimentos aos cursos d'água. Objetivou-se com este trabalho avaliar as fontes de sedimentos numa bacia hidrográfica rural de cabeceira com predomínio de cultivos anuais sob plantio direto e com intensa e inadequada exploração dos recursos naturais, por meio da quantificação da contribuição relativa das estradas e das lavouras na produção global de sedimentos. A bacia hidrográfica está localizada no município de Júlio de Castilhos, Rio Grande do Sul. O período de estudo foi de maio de 2009 a abril de 2011. Para a identificação das fontes, foi utilizado o método fingerprinting, que compara os solos de diferentes fontes e os sedimentos que são encontrados em suspensão no canal de drenagem, usando elementos traçadores. O manejo inadequado do solo nas áreas de lavoura, a falta de planejamento das vias de acesso e a ausência de práticas de controle do escoamento superficial, que sejam compatíveis com a fragilidade condicionada pelos solos e pelo relevo da bacia hidrográfica, têm provocado o surgimento de processos erosivos acelerados com efeitos negativos ao agricultor e à sociedade. As estradas apresentam alta porcentagem de contribuição na transferência de sedimentos, mas a contribuição das áreas de lavoura aumenta em precipitações pluviais de alta magnitude. Isso evidencia que a magnitude dos eventos de chuva

(1) Recebido para publicação em 22 de julho de 2013 e aprovado em 19 de novembro de 2013.

(2) Doutorando em Ciência do Solo, Universidade Federal de Santa Maria - UFSM. Av. Roraima, 1000, Bairro Camobi. CEP 97105900 Santa Maria (RS). Bolsista CAPES. E-mail: tales.t@mail.ufsm.br, andre.pellegrini@yahoo.com.br

(3) Professor do Departamento de Solos, UFSM. Bolsista do CNPq. E-mail: jminella@gmail.com, daniloesaf@gmail.com

(4) Professor Auxiliar do Departamento de Solos, Faculdade de Agronomia Eliseu Maciel, Universidade Federal de Pelotas. Campus Universitário, s/n. Caixa Postal 354. CEP 96010-900 Pelotas (RS). E-mail: tchemiguel@yahoo.com.br

(5) Doutorando em Ciência do Solo, UFSM. Bolsista CNPq. E-mail: jwrasche@yahoo.com.ar

(6) Doutoranda em Geografia, Universidade Federal do Paraná. Rua XV de Novembro, 1299, Bairro Centro. CEP 80060-000 Curitiba (PR). Bolsista CAPES. E-mail: capoane@gmail.com

(7) Aluno do Curso de Agronomia, UFSM. Bolsista CNPq. E-mail: lucasciotti@hotmail.com, gilmarschaefer2007@yahoo.com.br 


\begin{abstract}
influencia a proporção de contribuição entre as fontes ao longo do ano na bacia hidrográfica, interferindo no processo de mobilização de sedimentos e nutrientes em direção à rede de drenagem.
\end{abstract}

\title{
Termos de indexação: fingerprinting, elementos traçadores naturais, erosão
} hídrica.

\section{SUMMARY: THE CONTRIBUTION OF SEDIMENT SOURCES IN A RURAL CATCHMENT UNDER NO-TILLAGE}

\begin{abstract}
Knowledge of the main diffuse sources of sediment production can enhance efficiency in use of public resources invested in management strategies that seek to mitigate sediment transfer to waterways from catchment areas. The aim of the present study was to evaluate the sediment sources in a rural catchment with predominance of annual crops under a no-tillage system and with intense and inappropriate exploitation of natural resources (soil and water) by quantifying the relative contribution of roads and croplands in the overall production of sediments. Inappropriate exploitation is a result of a lack of planning of crop production areas and access pathways, both from the point of view of their location in the landscape, and of management practices which are incompatible with the conditions of soil fragility and relief. The catchment is located in the municipality of Julio de Castilhos, State of Rio Grande do Sul. The period under study was from May 2009 to April 2011. For identification of the sources we used the fingerprinting method, which compares the soils from different sources and the sediments in suspension in the drainage canal using tracer elements. Inadequate soil management in croplands, the lack of planning of access roads, and the absence of control practices for surface runoff compatible with the fragility brought about by the soil and topography of the watershed have caused the emergence of accelerated erosion with negative effects for the farmer and society. Roads represent a high percentage of contribution to sediment transfer, but the contribution of cropland increases with rainfall of high magnitude. This shows that the magnitude of rainfall events affects the proportion of contribution among sources throughout the year in the catchment area, affecting the process of mobilization of sediments and nutrients towards the drainage system.
\end{abstract}

Index terms: fingerprinting approach, natural tracer elements, water erosion.

\section{INTRODUÇÃO}

A criação do sistema plantio direto (SPD) foi uma das maiores evoluções da agricultura brasileira desde a Revolução Verde. O SPD é fundamentado em alguns princípios básicos como a redução ou eliminação do revolvimento do solo, a cobertura permanente do solo com resíduos vegetais ou plantas vivas pelo maior tempo possível e a diversificação de culturas, mediante o cultivo de múltiplas espécies, em rotação, sucessão e, ou, em consorciação de culturas, além de práticas mecânicas de conservação do solo. Esse sistema de manejo é sustentável e altamente produtivo, aumenta a estrutura, agregação e porosidade do solo, maximiza a ciclagem de nutrientes e estimula a atividade biológica do solo, além de proteger o solo contra os processos erosivos. Infelizmente, apenas uma fração das áreas cultivadas no Brasil segue todos os princípios fundamentais desse sistema conservacionista; via de regra, o único princípio utilizado massivamente pelos agricultores é o não revolvimento do solo com arações e gradagens, em razão principalmente das facilidades operacionais fruto do aprimoramento de implementos que possibilitaram a semeadura sem o revolvimento do solo. Após a consolidação do "plantio direto" (PD) mecanizado no Brasil, a área sem revolvimento do solo, incluindo áreas com cultivo sem revolvimento do solo ou "PD" e áreas sob SPD, para produção de culturas anuais, teve aumento exponencial de 1 milhão de hectares em 1992 para 25 milhões de hectares no final da primeira década do século XXI (Casão Junior et al., 2012), representando aproximadamente $25 \%$ da área sob PD no mundo (FEBRAPDP, 2011).

No Estado do Rio Grande do Sul, a área sob PD é de aproximadamente $90 \%$ da área cultivada. Grande parte dessa área é cultivada sem utilização de medidas de controle de escoamento superficial e sem sistemas de rotação de culturas. A inexistência de barreiras físicas e a falta de cobertura do solo acabam potencializando as perdas de solo nesse sistema, principalmente pela erosão em entressulco, em sulcos e em canal. Além disso, sistemas de integração lavoura-pecuária sob PD com manejo inadequado provocam adensamento da camada superficial do solo e, ou, diminuem a cobertura do solo para o cultivo de verão (Souza et al., 2010), facilitando o escoamento superficial de água e os processos erosivos. Como resultado do manejo inadequado das áreas de lavouras 
sob PD, as estradas rurais acabam atuando como canal escoador do excesso de água proveniente das lavouras. Além disso, a maioria das estradas em bacias hidrográficas rurais é alocada sem planejamento na paisagem, no sentindo do declive; essas não são pavimentadas e não apresentam estruturas de contenção da enxurrada, tornando-se também importante fonte de sedimentos. Esse panorama representa grande porção da área agrícola do RS. No entanto, os estudos hidrossedimentológicos e de identificação da contribuição das fontes difusas na produção global de sedimentos ainda são incipientes nessas áreas.

O transporte fluvial de sedimentos em suspensão gera inúmeros problemas como assoreamento de barragens, reservatórios e canais, transporte de nutrientes e contaminantes e degradação dos ambientes aquáticos. A erosão observada nas lavouras e nas estradas está relacionada com os impactos observados nos rios, tanto sob o aspecto físico como químico e biológico, mas a conectividade entre esses dois fenômenos não é direta, pois é regida por mecanismos complexos resultantes do comportamento hidrossedimentológico de cada bacia hidrográfica (Minella \& Merten, 2011). Por isso, uma das principais limitações da maioria dos estudos de transferência de sedimentos, sejam eles quantitativos ou qualitativos, é a falta de informação sobre a origem dos sedimentos (Collins \& Walling, 2004). O conhecimento do padrão espacial das principais fontes de poluição difusa, como os sedimentos, pode aumentar a eficiência de utilização dos recursos investidos em estratégias de gestão que visem mitigar a transferência de sedimentos e poluentes neles adsorvidos aos cursos d'água. Por isso, nos últimos anos, houve interesse crescente em estudos, objetivando compreender o padrão espacial das fontes de sedimentos em suspensão a fim de contribuir para a descrição dos processos de ligação entre calha e vertente, bem como para o planejamento dos recursos naturais.

A contribuição das fontes de sedimentos é estimada por métodos diretos e indiretos. Os métodos mais comuns são os indiretos, que envolvem observações visuais, por exemplo, pela análise de fotos aéreas (Day et al., 2013) ou medidas de atividade erosiva, como com parcelas de erosão (Reichert et al., 2001). Os métodos diretos de estimativa da contribuição das fontes de sedimento baseiam-se no fato de que as características físicas e químicas dos sedimentos erodidos guardam relação com as características dos solos nas principais fontes de sedimentos. O método emprega características traçantes naturais (passivas), como cor (Krein et al., 2003; Martínez-Carreras et al., 2010a,b,c), suscetibilidade magnética (Caitcheon, 1993; Slattery et al., 1995; Owens \& Walling, 2002), mineralogia (Motha et al., 2003; Wu et al., 2011), atividade enzimática (Nosrati et al., 2011), isótopos ${ }^{87} \mathrm{Sr} / 86 \mathrm{Sr}$ - Smith et al., $2009 ;{ }^{239} \mathrm{Pu}$ e ${ }^{240} \mathrm{Pu}$ - Lindahl et al., 2010; ${ }^{206} \mathrm{~Pb},{ }^{207} \mathrm{~Pb},{ }^{208} \mathrm{~Pb}$ - Bird et al., 2010) e composição geoquímica (Minella et al., 2008; Collins et al., 2010a,b,c; Collins et al., 2012a,b), ou mesmo utilizando elementos artificiais (propriedades ativas) como os radionuclídeos ${ }^{137} \mathrm{Cs}$, ${ }^{7} \mathrm{Be}$ e ${ }^{210} \mathrm{~Pb}$ (Walling et al., 2008; Du et al., 2010; Estrany et al., 2010) e até mesmo pela adição de partículas fluorescentes artificiais (Granger et al., 2011). Todavia, a utilização de poucos elementos traçadores gera muita incerteza matemática na determinação das fontes, especialmente em estudos envolvendo um número grande de fontes de sedimentos em potencial (Collins \& Walling, 2002). Nesse sentido, Yu \& Oldfield (1989) demonstraram que a resolução quantitativa de múltiplas fontes de sedimentos pode ser alcançada utilizando assinaturas compostas (composed fingerprints), envolvendo vários traçadores considerados estatisticamente independentes. Consequentemente, considera-se que cálculos quantitativos para estimativa da proporção de contribuição das fontes são mais úteis do que descrições puramente qualitativas (Collins et al., 1997).

Objetivou-se com este trabalho avaliar as fontes de sedimentos numa bacia hidrográfica rural de cabeceira com predomínio de cultivos anuais sob plantio direto com intensa e inadequada exploração dos recursos naturais, por meio da quantificação da contribuição relativa das estradas e das lavouras na produção global de sedimentos.

\section{MATERIAL E MÉTODOS}

\section{Caracterização da área de estudo}

A bacia hidrográfica $(\mathrm{BH})$ pesquisada neste estudo está localizada no Assentamento Alvorada, no município de Júlio de Castilhos, Região Geomorfológica Planalto das Missões do Estado do Rio Grande do Sul (Robaina et al., 2010). As águas da BH do assentamento Alvorada drenam para a bacia hidrográfica do Alto Jacuí. A hierarquia fluvial na BH seguindo os critérios introduzidos por Strahler (1957) é de terceira ordem, ou seja, canais que se originam da confluência de dois canais de segunda ordem, podendo receber afluentes de segunda e primeira ordens. A BH apresenta fator forma de 0,47 e coeficiente de compacidade de 1,34. O tempo médio de concentração e o tempo médio de pico são 82 e 210 min, respectivamente. Conforme Rossato (2011), o clima da região corresponde ao subtropical medianamente úmido com variação longitudinal das temperaturas médias. A temperatura média anual varia entre $17-20^{\circ} \mathrm{C}$. A temperatura média do mês mais frio oscila entre $11-14^{\circ} \mathrm{C}$, e a temperatura média do mês mais quente varia entre $23-26{ }^{\circ} \mathrm{C}$. A precipitação pluvial oscila entre $1500-1700 \mathrm{~mm}$ ao ano em 90-110 dias de chuva. A BH possui $0,783 \mathrm{~km}^{2}$ (Figura 1), com relevo homogêneo, formado de modo geral por colinas suaves (declividade média de $0,07 \mathrm{~m} \mathrm{~m}^{-1}$ ), bem arredondadas, esculpidas em rochas vulcânicas básicas da Formação Serra Geral e, em 


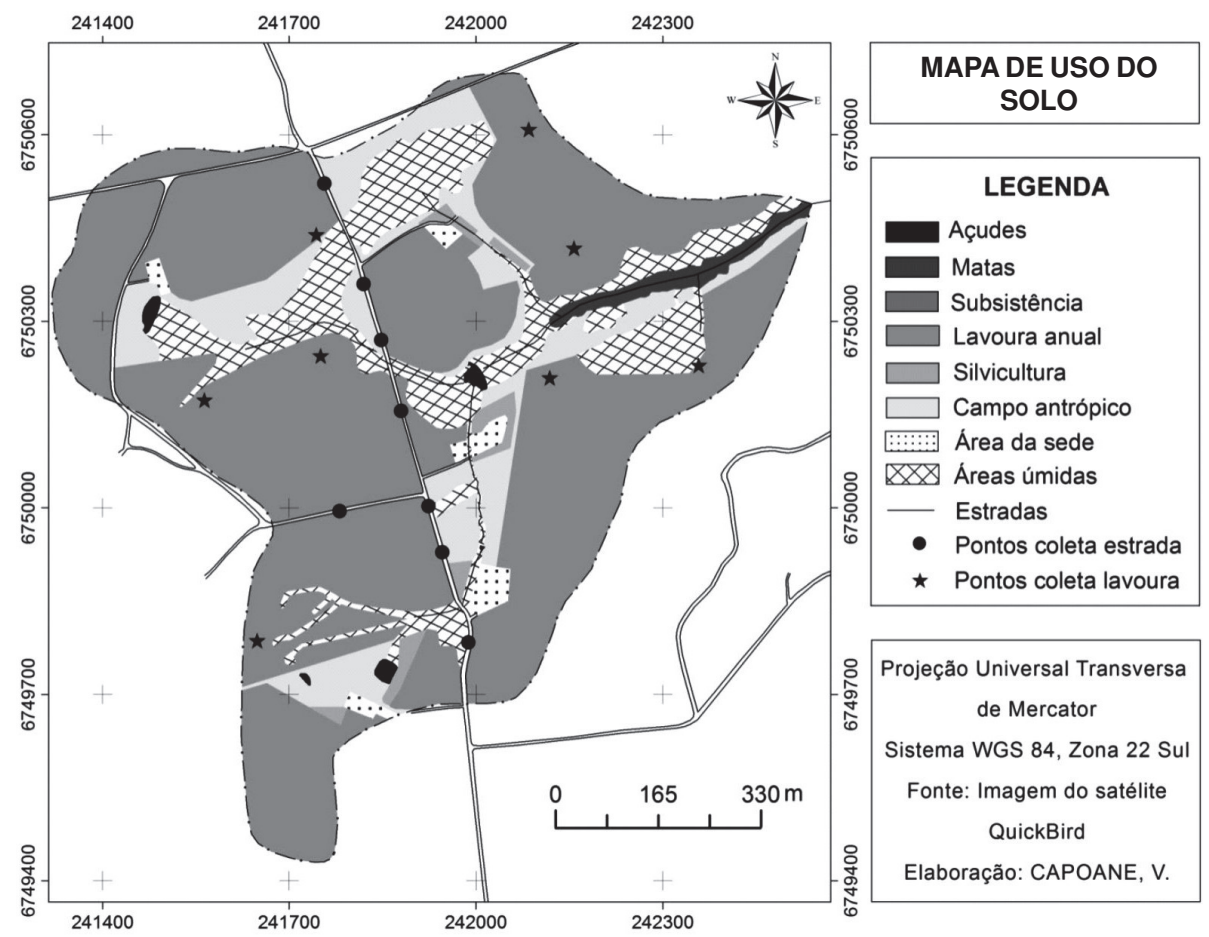

Figura 1. Mapa de uso do solo na bacia hidrográfica do Assentamento Alvorada, Júlio de Castilhos, Rio Grande do Sul.

menores proporções, em rochas sedimentares correspondentes à Formação Tupanciretã (IBGE, 1986). A classe de solo predominante é a dos Argissolos, com pequena proporção de Cambissolos e Neossolos, podendo ainda ser encontrada em locais planos e úmidos, a classe dos Gleissolos. A textura média de 20 amostras de solos contemplando toda a variabilidade de solos presentes na BH para a camada de $0-20 \mathrm{~cm}$ foi de $67,2 \pm 5,3 \%$ de areia, $17,4 \pm 3,4 \%$ de silte e 15,4 $\pm 3,9 \%$ de argila.

O principal uso do solo na BH é o cultivo de plantas anuais (51,7 ha, 66,1 \% área total) (Figura 1). Nessas áreas, faz-se o cultivo de soja ou milho sem o revolvimento do solo no verão e pastoreio de aveia e azevém, no inverno. Um dos problemas mais graves da BH é a erosão em entressulco e a erosão em sulco em razão da baixa adoção de sistemas de manejo conservacionistas, caracterizada pela ausência de práticas de controle de escoamento superficial, incipiente rotação de culturas e com insuficiente cobertura do solo e aporte de biomassa. Além disso, na implantação das pastagens de inverno, uma prática comum entre os agricultores é a semeadura a lanço com posterior incorporação por meio de uma gradagem, que acaba mobilizando a camada superficial do solo, tornando-o mais susceptível aos processos erosivos.

As estradas ocupam 1,5\% da área total da bacia hidrográfica (Figura 1). As principais características das vias de acesso que favorecem o processo erosivo na escala de bacia são: estradas não pavimentadas, alocação na paisagem sem planejamento, ausência de estruturas de contenção da enxurrada e o fato de elas serem utilizadas como canal escoador do excesso de água provenientes das lavouras. Consequentemente, é possível verificar grandes sulcos e canais de erosão com grande perda pelo escoamento concentrado. Para manter a trafegabilidade das estradas presentes na $\mathrm{BH}$, é adicionada frequentemente uma espessa camada (entre 10-20 cm) de material alóctone rochoso. Esse material é o horizonte $\mathrm{C}$ do solo (arenito) de uma área contígua a BH (aproximadamente $300 \mathrm{~m}$ ) que também é erodido e mobilizado em direção à rede de drenagem. Sendo assim, nota-se claramente que as estradas assumem papel importante no regime hidrológico e sedimentológico da bacia.

\section{Amostragem do solo das fontes e dos sedimentos em suspensão}

A coleta das amostras nos solos com evidências de erosão (fontes potenciais de sedimentos) foi realizada com base no mapa de uso e ocupação do solo e em observações do processo de remoção e transporte e mobilização de sedimentos no campo. As principais fontes de sedimentos selecionadas foram lavouras anuais e estradas. Nos outros usos do solo (pastagens permanentes, mata e silvicultura), não foram identificados evidências significativas do processo erosivo. Para realizar uma amostragem representativa da variabilidade das estradas e das áreas de lavoura, foram coletadas oito amostras por fonte; cada amostra foi composta por 10 subamostras na profundidade de $0-5 \mathrm{~cm}$. 
O sedimento em suspensão foi coletado no exutório da $\mathrm{BH}$ com um amostrador integrador de sedimentos em suspensão no tempo (Phillips et al., 2000). Esse coletor possui um orifício de $4 \mathrm{~mm}$ de diâmetro que permite a passagem do fluxo de água e outro que permite a saída. Com base no princípio de sedimentação, o material sólido presente no escoamento entra no amostrador, e pela baixa velocidade do fluxo no interior dele ocorre a sedimentação e a retenção do material fino, integrando em uma única amostra o sedimento em suspensão que passa por determinado período de tempo. Foram realizadas quatro coletas de sedimento em suspensão, compreendendo os períodos de 11 de maio de 2009 a 28 de outubro de 2009,28 de outubro de 2009 a 16 de julho de 2010, 16 de julho de 2010 a 11 de outubro de 2010 e 11 de outubro de 2010 a 17 de abril de 2011, representando um período de $170,261,87$ e 188 dias, respectivamente. A estratégia de coleta dessas quatro amostras em épocas distintas foi: obter repetição nas amostras a serem analisadas e verificar a variabilidade intra-anual e interanual do padrão de emissão das fontes.

As amostras de solo das fontes e de sedimentos em suspensão foram secas em estufa de circulação forçada de ar a $\pm 50^{\circ} \mathrm{C}$. Posteriormente, foram peneirados em malha de $150 \mu \mathrm{m}$. Esse limite foi escolhido para diminuir as incertezas relacionadas com o efeito de diluição das partículas de areia e considerar os agregados de argila que são transportados em suspensão. Esse limite foi estabelecido a partir da observação da granulometria do material transportado em suspensão, que apresentou mais de $90 \%$ das partículas com diâmetro inferior a $150 \mu \mathrm{m}$ (D90).

\section{Análises do solo das fontes e dos sedimentos em suspensão}

Após o peneiramento, 0,5 g das amostras de solo das fontes e de sedimentos em suspensão foram digeridas com peróxido de hidrogênio $\left(\mathrm{H}_{2} \mathrm{O}_{2}\right)+$ ácido fluorídrico $(\mathrm{HF})+$ ácido perclórico $\left(\mathrm{HClO}_{4}\right)+$ ácido clorídrico $(\mathrm{HCl})$ a $100{ }^{\circ} \mathrm{C}$. Posteriormente, os teores totais de $\mathrm{Ca}, \mathrm{Cu}, \mathrm{Fe}, \mathrm{Mg}, \mathrm{Mn}$, e $\mathrm{Zn}$ nos extratos das digestões foram determinados por espectrometria de absorção atômica (EAA); o teor de P total, colorimetricamente pelo método de Murphy \& Riley (1962); e os teores de $\mathrm{K} \mathrm{e} \mathrm{Na}$, por espectrometria de emissão de chama.

Foram estimados também os teores de óxidos cristalinos de $\mathrm{Fe}$ e $\mathrm{Mn}$ por extração com ditionitocitrato-bicarbonato de sódio $\left(\mathrm{Fe}_{\mathrm{d}}\right.$ e $\left.\mathrm{Mn}_{\mathrm{d}}\right)$, segundo método da Embrapa (1997) adaptado de Mehra \& Jackson (1960), e os teores de óxidos amorfos ou de baixa cristalinidade de $\mathrm{Fe}$ e $\mathrm{Mn}$, pela extração com oxalato ácido de amônio $\left(\mathrm{Fe}_{0}\right.$ e $\left.\mathrm{Mn}_{0}\right)$ (Embrapa, 1997).

\section{Análises estatísticas}

A primeira etapa da análise estatística foi realizada para estabelecer o conjunto de variáveis com capacidade de discriminar as fontes, por meio da aplicação de dois testes sequenciais: o teste de comparação de médias não paramétrico de MannWhitney $(U)$; e uma função discriminante multivariada. O teste $U$ permite testar a hipótese nula de que as fontes são provenientes de uma mesma população. Neste teste, foram definidos os elementos químicos que apresentam diferença estatística entre as fontes de sedimentos, podendo ser, dessa forma, utilizados como variáveis traçadoras (Walling \& Collins, 2000). O teste foi aplicado para cada variável, verificando sua capacidade individual em discriminar as fontes.

A função discriminante multivariada determina o número mínimo de variáveis que maximiza a discriminação das fontes. Nesse teste, foram utilizadas apenas as variáveis que apresentaram diferenças entre as fontes. Ele é baseado no parâmetro Wilks' Lambda $\left(\Lambda^{*}\right)$ da análise de variância e o critério utilizado pelo modelo estatístico é a minimização de $\Lambda^{*}$ (Johnson \& Wichern, 1992). Dessa forma, quando $\Lambda^{*}$ for próximo de 1 , significa que não existe capacidade discriminante (as fontes são consideradas iguais) do conjunto de variáveis selecionadas. Já valores próximos de 0 ocorrem quando a variabilidade dentro do grupo é pequena em comparação com a variabilidade total, isto é, a maior parte da variabilidade total poderá ser atribuída à diferença entre os grupos.

Após a definição do conjunto de variáveis pela minimização de $\Lambda^{*}$, foi determinada a proporção de cada fonte na composição do sedimento suspendido. Na equação 1, descreve-se a relação matemática entre as proporções das fontes e as variáveis das fontes e dos sedimentos em suspensão (Walling \& Woodward, 1995).

$$
\mathrm{y}_{\mathrm{i}}=\sum_{\mathrm{s}=1}^{\mathrm{n}} \mathrm{a}_{\mathrm{is}} \mathrm{P}_{\mathrm{s}}(\mathrm{s}=1,2, \ldots, \mathrm{n}) \mathrm{e}(\mathrm{i}=1,2, \ldots, \mathrm{m})
$$

em que $y_{i}$ é o valor da variável $i$ obtida no sedimento em suspensão, $a_{i s}$ são os coeficientes do modelo linear (concentração do elemento químico $i$ na fonte s) e $P_{s}$ é a proporção da massa da fonte $s$, que podem ser apresentadas como um conjunto de funções lineares de $m$ variáveis e $n$ fontes.

Para determinar os valores de $P_{s}$, foi utilizada uma função objetivo (Walling \& Woodward, 1995). A solução foi encontrada por meio de um processo iterativo objetivando minimizar o valor de $R$ da equação 2 , utilizando-se a função $f$ mincon no programa Matlabß . No processo de minimização, os valores de $P$ foram sujeitos a duas restrições: serem maiores ou iguais a zero e menores ou iguais a um (Equação 3); e a soma dos $P$ deve ser igual a um (Equação 4$)$.

$$
\begin{gathered}
R=\sum_{s=1}^{m}\left\{\frac{y_{i-}\left(\sum_{s=1}^{n} P_{s} a_{i s}\right)}{y_{i}}\right\}^{2} \\
0 \leq P_{s} \leq 1
\end{gathered}
$$




$$
\sum_{\mathrm{s}=1}^{\mathrm{g}} \mathrm{P}_{\mathrm{s}}=1
$$

em que $m$ é o número de elementos químicos selecionados como variáveis traçadoras; $n$, o número de fontes; $y_{i}$, a concentração da variável traçadora $i$ na amostra de sedimento em suspensão; $P_{s}$, a proporção de contribuição da fonte $s ; a_{i s}$, o valor médio da variável traçadora $i$ obtido na fonte $s$. Esse modelo (Equações 2, 3 e 4) foi executado no programa Matlabß.

Por fim, foi avaliado o processo de otimização da equação 4, comparando a concentração química dos elementos utilizados (variáveis traçadoras) nos sedimentos em suspensão, e o valor predito pelo modelo, com base na proporção calculada para cada fonte. Posteriormente, com os valores do erro relativo de cada variável, foi calculado um erro relativo médio (ERM) para providenciar um valor único associado a cada amostra de sedimentos em suspensão (Equação 5).

$$
\mathrm{ERM}=\sum_{\mathrm{i}=1}^{\mathrm{m}}\left\{\frac{\mathrm{y}_{\mathrm{i}}-\left(\sum_{\mathrm{s}=1}^{\mathrm{n}} \mathrm{P}_{\mathrm{s}} \mathrm{a}_{\text {is }}\right)}{\mathrm{m}}\right\}
$$

Valores menores que $15 \%$ para a equação 5 indicam que o modelo encontrou uma solução viável dos valores de $P_{s}$ (contribuições relativas de cada fonte), proveniente do procedimento de minimização da equação 1 (Walling \& Collins, 2000).

\section{RESULTADOS E DISCUSSÃO}

\section{Descrição do potencial discriminante das fontes}

Das 13 variáveis avaliadas no solo das fontes, 10 foram selecionas utilizando-se um nível de significância de $90 \%\left(U_{\text {crítico }}=2.7055\right)$ pela aplicação do teste de Mann-Whitney (Quadro 1). Apesar de ser uma quantidade relativamente baixa de variáveis para estudos dessa natureza, foi verificado que esse conjunto representa processos antrópicos e geomorfológicos distintos e, por essa razão, considerou-se que foi suficiente para discriminar as fontes potenciais de erosão selecionadas. Nesse sentido, dentre as variáveis utilizadas para discriminar as fontes, algumas refletem práticas agrícolas realizadas nas áreas de lavoura da $\mathrm{BH}$, como o $\mathrm{P}$ e o Ca. Esses dois nutrientes apresentaram maiores teores nas áreas de lavoura do que na estrada. O maior teor de $\mathrm{P}$ resulta de vários anos de cultivo das áreas de lavoura com aplicação de fertilizantes fosfatados (Tiecher et al., 2012). Já o maior teor de Ca nas áreas de lavoura resulta das aplicações de calcário realizadas nessas áreas, com o objetivo de aumentar o pH do solo e neutralizar o Al tóxico do solo que prejudica o crescimento e desenvolvimento das culturas anuais cultivadas na BH (Kaminski et al., 2005).
O K também é um nutriente amplamente aplicado via fertilizantes nas lavouras. Contudo, o teor de $\mathrm{K}$ total no solo da estrada foi muito maior que o no solo das lavouras, 4.150 e $746 \mathrm{mg} \mathrm{kg}^{-1}$, respectivamente. Isso ocorreu em razão de o material alóctone depositado nas estradas (arenito), possuir grande quantidades de minerais menos intemperizados como feldspatos potássicos $\left(\mathrm{KAlSi}_{3} \mathrm{O}_{8}\right)$, que possuem altos teores de $\mathrm{K}$. Além disso, como esse material não é tão intemperizado como o solo das lavouras da BH, ele possui também alto teor de bases como o $\mathrm{Na}$ e $\mathrm{Mg}$, o que resultou nos teores mais elevados desses nutrientes na estrada do que na lavoura (Quadro 1). É também indício de que o material encontrado nas estradas possui menor intemperismo do que o solo da lavoura a relação entre o teor de $\mathrm{Fe}_{\mathrm{d}}$ e o teor de Fe total $\left(\mathrm{Fe}_{\mathrm{d}} / \mathrm{Fe}_{\text {total }}\right)$. Quanto maior essa relação tanto mais intemperizado é o solo e menor o teor de Fe contido em minerais primários. Dessa forma, verificou-se que a relação encontrada nas estradas foi de $0,514 \pm 0,119$ e nas lavouras, de $0,679 \pm 0,080$. Durante o intemperismo, ocorre maior formação de óxidos de Fe mal cristalizados ou amorfos, estimados pelos teores de $\mathrm{Fe}_{0}$, e por isso o teor de $\mathrm{Fe}_{0}$ e inclusive a relação $\mathrm{Fe}_{\mathrm{o}} / \mathrm{Fe}_{\mathrm{d}}$ foram maiores no solo das lavouras.

A aplicação da função discriminante forneceu um valor de Wilk's Lambda de 0,040 com oito (P, Fe, Zn, $\mathrm{K}, \mathrm{Mg}, \mathrm{Mn}, \mathrm{Ca}, \mathrm{Na}$ ) das 10 variáveis selecionadas pelo teste de Mann-Whitney (Quadro 2). Como o valor de $\Lambda^{*}$ é a proporção da variabilidade total das fontes por causa do erro, o conjunto das oito variáveis selecionadas forneceu um erro de 4,0 \%; ou seja, o conjunto das variáveis selecionadas explica, aproximadamente, 96,0 \% das diferenças entre as fontes. Mesmo assim, todas as amostras foram corretamente classificadas nas respectivas fontes onde foram coletadas (Quadro 2).

\section{Classificação das amostras de sedimentos em suspensão}

O modelo de classificação das amostras de sedimento em suspensão, que utiliza a minimização de uma função objetivo (Equação 2), foi eficiente na determinação das contribuições das fontes nos quatro períodos de avaliação, pois os erros médios foram inferiores a $15 \%$ (Figura 2b). A contribuição relativa das estradas na produção de sedimentos variou de 42,7 a 69,2 \%, e a contribuição das áreas de lavoura, de 30,8 a $57,3 \%$.

Os efeitos de preparo do solo apresentam fortes evidências de erosão na bacia. No outono, são implantadas as pastagens de inverno em 66,1 \% da área total. Na maior parte dessa área, a semeadura é realizada a lanço, com posterior incorporação das sementes com gradagem, mobilizando a camada superficial do solo, tornando-a mais susceptível aos processos erosivos. Todavia, não foi observada relação entre a variação da contribuição das fontes de sedimentos e as práticas realizadas. Isso pode ter 
Quadro 1. Resultados do teste de Mann-Whitney para cada variável e teor de cada variável no solo das estradas e das lavouras

\begin{tabular}{|c|c|c|c|c|c|}
\hline \multirow{2}{*}{ Variável } & \multicolumn{2}{|c|}{ Teste Mann-Whitney } & \multirow{2}{*}{ Amostras classificadas corretamente } & \multirow{2}{*}{$\frac{\text { Estrada }}{\text { Média } \pm \mathrm{DP}^{(4)}}$} & \multirow{2}{*}{$\frac{\text { Lavoura }}{\text { Média } \pm \text { DF }}$} \\
\hline & $U^{(1)}$ & $\mathbf{p}^{(2)}$ & & & \\
\hline & & & $\%$ & & \\
\hline \multicolumn{6}{|l|}{ Teor total } \\
\hline $\mathrm{P}\left(\mathrm{mg} \mathrm{kg}^{-1}\right)$ & 11,294 & $<0,001^{*}$ & 93,75 & $170 \pm 63$ & $342 \pm 42$ \\
\hline $\mathrm{K}\left(\mathrm{mg} \mathrm{kg}^{-1}\right)$ & 3,579 & $0,059^{*}$ & 87,50 & $4150 \pm 2558$ & $746 \pm 101$ \\
\hline $\mathrm{Na}\left(\mathrm{mg} \mathrm{kg}{ }^{-1}\right)$ & 4,451 & $0,035^{*}$ & 87,50 & $2764 \pm 1462$ & $859 \pm 204$ \\
\hline $\mathrm{Ca}\left(\mathrm{mg} \mathrm{kg}{ }^{-1}\right)$ & 4,864 & $0,027^{*}$ & 87,50 & $185 \pm 103$ & $427 \pm 278$ \\
\hline $\mathrm{Mg}\left(\mathrm{mg} \mathrm{kg}^{-1}\right)$ & 5,835 & $0,016^{*}$ & 75,00 & $2298 \pm 359$ & $1619 \pm 556$ \\
\hline $\mathrm{Cu}\left(\mathrm{mg} \mathrm{kg}{ }^{-1}\right)$ & 11,344 & $<0,001^{*}$ & 75,00 & $40,0 \pm 5,0$ & $21,3 \pm 8,6$ \\
\hline $\mathrm{Zn}\left(\mathrm{mg} \mathrm{kg}^{-1}\right)$ & 5,338 & $0,021^{*}$ & 75,00 & $47,1 \pm 8,6$ & $34,9 \pm 7,9$ \\
\hline $\mathrm{Fe}\left(\mathrm{mg} \mathrm{kg} \mathrm{kg}^{-1}\right)$ & 11,294 & $<0,001^{*}$ & 100,00 & $66214 \pm 18555$ & $34786 \pm 8863$ \\
\hline $\operatorname{Mn}\left(\mathrm{mg} \mathrm{kg}^{-1}\right)$ & 6,333 & $0,012^{*}$ & 56,25 & $489 \pm 261$ & $234 \pm 54$ \\
\hline \multicolumn{6}{|l|}{ Teor de óxidos } \\
\hline $\mathrm{Fe}_{\mathrm{d}}\left(\mathrm{mg} \mathrm{kg}{ }^{-1}\right)$ & 2,165 & 0,141 & 93,75 & $35903 \pm 19696$ & $23460 \pm 6350$ \\
\hline $\mathrm{Fe}_{\mathrm{o}}\left(\mathrm{mg} \mathrm{kg}{ }^{-1}\right)$ & 8,660 & $0,003^{*}$ & 81,25 & $793 \pm 337$ & $1,699 \pm 439$ \\
\hline $\mathrm{Mn}_{\mathrm{d}}\left(\mathrm{mg} \mathrm{kg} \mathrm{kg}^{-1}\right)$ & 1,588 & 0,208 & 56,25 & $329 \pm 287$ & $187 \pm 71$ \\
\hline $\mathrm{Mn}_{\mathrm{o}}\left(\mathrm{mg} \mathrm{kg}^{-1}\right)$ & 0,044 & 0,833 & 50,00 & $244 \pm 236$ & $180 \pm 71$ \\
\hline $\mathrm{Fe}_{\mathrm{d}} / \mathrm{Fe}_{\text {total }}{ }^{(3)}$ & 6,353 & $0,012^{*}$ & - & $0,514 \pm 0,119$ & $0,679 \pm 0,080$ \\
\hline $\mathrm{Fe}_{\mathrm{o}} / \mathrm{Fe}_{\mathrm{d}}^{(3)}$ & 11,294 & $<0,001^{*}$ & - & $0,023 \pm 0,004$ & $0,080 \pm 0,037$ \\
\hline
\end{tabular}

(1) Valor do teste de discriminação que se aproxima dos valores da distribuição qui-quadrado - $U_{\text {crítico }}(90 \%$; e 1 gl) $=2,7055$. (2) Probabilidade de que as fontes difiram entre si, segundo a distribuição qui-quadrado; * valores de p<0,10. ${ }^{(3)}$ Os valores das relações $\mathrm{Fe}_{\mathrm{d}} / \mathrm{Fe}_{\text {total }}$ e $\mathrm{Fe}_{\mathrm{o}} / \mathrm{Fe}_{\mathrm{d}}$ não foram utilizados como variáveis traçadoras por não serem linearmente aditivas (Walling, 2005; D'Haen et al., 2012). ${ }^{(4)}$ DP: desvio padrão.

Quadro 2. Resultado da análise da função discriminante com acréscimo gradual de cada variável e os valores correspondentes da minimização do Wilk's Lambda $\left(\Lambda^{*}\right)$

\begin{tabular}{cccc}
\hline Step & Variável & $\boldsymbol{\Lambda}^{*}$ acumulado $^{(1)}$ & Amostras classificadas corretamente $^{\circ}$ \\
\hline & & & $\%$ \\
2 & $\mathrm{P}$ & 0,255 & 97,5 \\
3 & $\mathrm{Fe}$ & 0,071 & 100,0 \\
4 & $\mathrm{Zn}$ & 0,066 & 100,0 \\
5 & $\mathrm{~K}$ & 0,062 & 100,0 \\
6 & $\mathrm{Mg}$ & 0,057 & 100,0 \\
7 & $\mathrm{Mn}$ & 0,050 & 100,0 \\
8 & $\mathrm{Ca}$ & 0,041 & 100,0 \\
\hline
\end{tabular}

(1) Valores acumulados com a adição de cada variável.

ocorrido em parte em razão dos longos e irregulares intervalos de coletas de sedimento em suspensão, mas demonstra também que outros fatores, além das práticas agrícolas, são preponderantes no controle da produção e emissão de sedimentos ao longo do ano na $\mathrm{BH}$ estudada, especialmente a conectividade das encostas com a rede de drenagem e as variações no regime pluviométrico.

Como se pode verificar na figura $2 \mathrm{a}$, a precipitação pluvial média histórica é bem distribuída ao longo do ano na BH. Apesar de o mês de setembro de 2009 ter registrado uma precipitação 129,7 \% maior que a média histórica, durante o primeiro período de coleta de sedimentos em suspensão, a precipitação ocorrida foi semelhante à média histórica da região; a variação na precipitação total para o período foi de $-7,2 \%$, em razão dos meses de junho e julho de 2009 que registraram precipitações 50,6 \% menores que a média histórica. Dessa forma, pôde-se concluir que a estrada é a principal fonte de sedimentos quando ocorre regime pluviométrico em eventos de pequena e média magnitude (69,2 \% da produção global de sedimentos). 


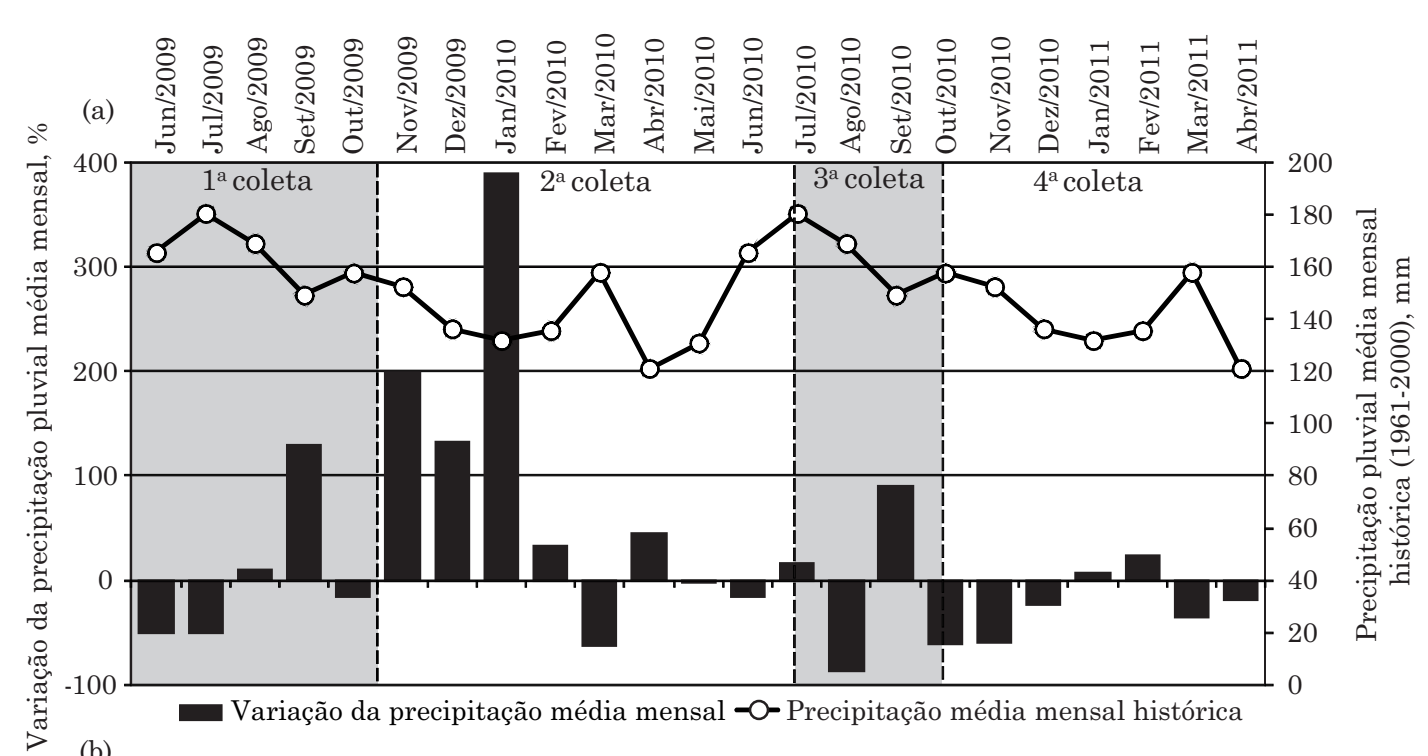

(b)

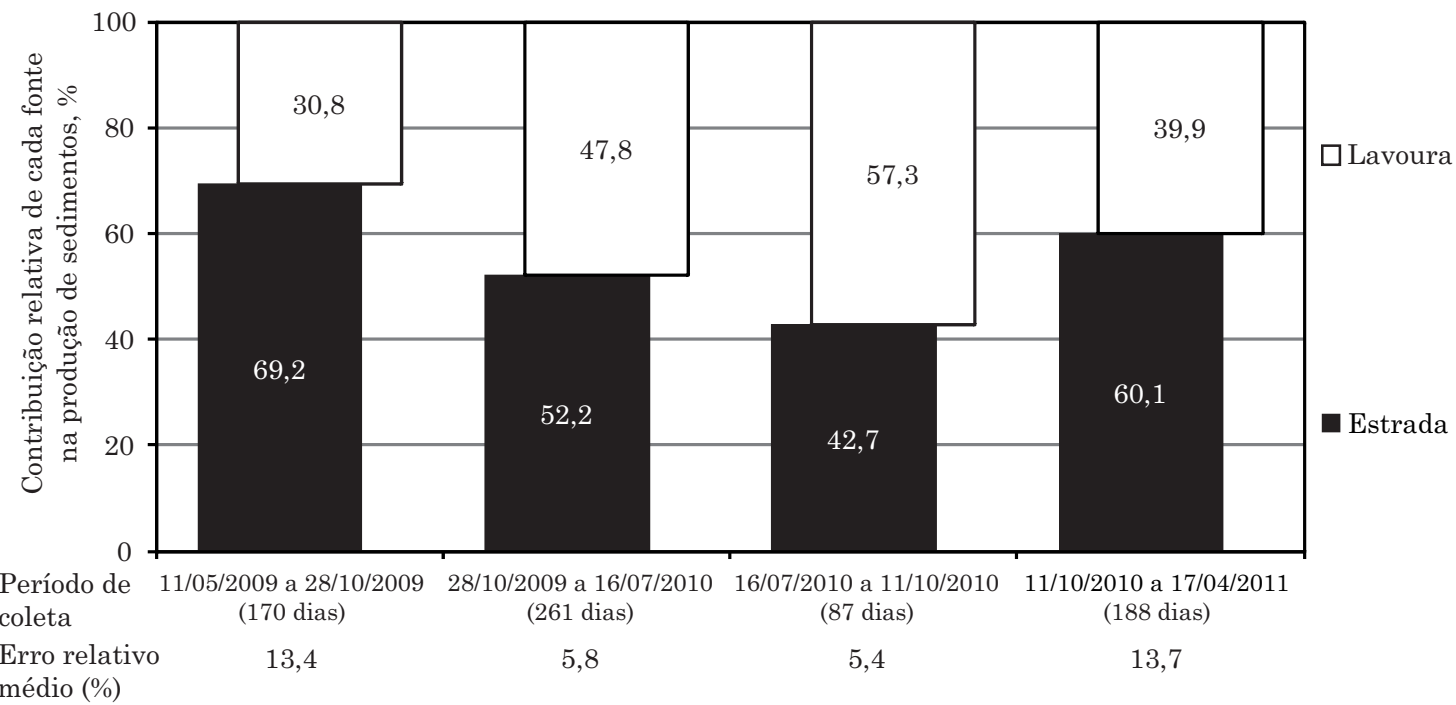

Figura 2. a) variação da precipitação pluvial média mensal durante os períodos de coleta de sedimento em suspensão (relação entre precipitação média mensal histórica e precipitação mensal durante o período de estudo) e precipitação pluvial média mensal histórica (1961-2000); e b) Contribuição relativa das áreas de lavoura e da estrada na produção de sedimentos na bacia hidrográfica do Assentamento Alvorada, Júlio de Castilhos, Rio Grande do Sul.

O tráfego intenso nas estradas diminui drasticamente a capacidade de infiltração do solo, onde o escoamento superficial da água e a erosão do solo podem ocorrer mesmo em eventos pluviométricos de baixa intensidade. Além dos aspectos negativos da rede viária descritos anteriormente, a grande contribuição das estradas à produção global de sedimentos deve-se também à composição inadequada do material utilizado para dar maior trafegabilidade (elevada quantidade de areia fina e baixa quantidade de argila), baixa frequência de manutenção, ligação direta entre as estradas e rede fluvial e entrada de escoamento provenientes das lavouras.

No segundo período de coleta, a precipitação pluvial na $\mathrm{BH}$ foi muito acima da média histórica (1,76 vez), especialmente durante os meses de novembro e dezembro de 2009 e janeiro de 2010, por causa das manifestações do fenômeno climático El Niño. Com a maior precipitação, o solo das lavouras pode ter atingido condições de alta umidade durante um período de tempo maior que o normal. Dessa forma, durante eventos de maior intensidade, a capacidade de infiltração do solo das áreas de lavouras pode ter sido excedida, fato esse que, associado à baixa adoção de sistemas de manejo conservacionistas, como terraços e plantio em nível, e ao uso de $\mathrm{PD}$ sem rotação de culturas e com pouquíssima cobertura do solo, resulta no aumento da contribuição das áreas de lavoura na produção global de sedimentos de 30,8 para $47,8 \%$ (Figura 2b). 
No terceiro período de coleta, a precipitação ocorrida voltou a ser semelhante à média histórica da região; a variação na precipitação total foi de apenas $-3,5 \%$. Apesar disso, a contribuição das áreas de lavoura manteve-se alta (57,3\%). Isso pode ter ocorrido porque grande parte dos sedimentos transportados das áreas de lavouras durante o segundo período de coleta pode ter sido depositada ao longo da $\mathrm{BH}$. Posteriormente, esse material continuou sendo exaurido da $\mathrm{BH}$ durante o terceiro período de coleta, resultando em elevada contribuição das áreas de lavouras na produção global de sedimentos. Em seguida, durante o quarto período, a precipitação ocorrida voltou a ser semelhante à média histórica da região (Figura 4) e, dessa forma, a contribuição das estradas na produção global de sedimentos voltou a prevalecer sob as áreas de lavouras (Figura 2b).

Verificou-se que a baixa adoção de sistemas de manejo conservacionistas como terraços, plantio em nível, rotação de culturas e cobertura do solo nas áreas de lavoura da $\mathrm{BH}$ potencializou a emissão de sedimentos dessas áreas em condições de excesso de chuvas. Além disso, uma vez que as estradas representam um componente estático da paisagem agrícola numa bacia hidrográfica, e dado a importância de sua contribuição na geração de sedimentos, o planejamento de alocação delas é primordial em programas de mitigação de transferência de sedimentos (Collins et al., 2010c). Para a melhor compreensão dos processos erosivos na BH estudada, os futuros trabalhos devem contemplar outras fontes potenciais de sedimento, como a própria rede fluvial e as áreas sob pastagens permanentes. Esse último uso, apesar de não possuir evidências fortes de erosão, ocupa uma área significativa da BH. Entendeu-se também que os açudes podem estar retendo parte do sedimento erodido das áreas de lavoura na $\mathrm{BH}$, gerando fonte de incerteza que potencializa a contribuição das estradas, que na sua maioria possui ligação direta com a rede de drenagem. Nesse sentido, é de grande importância que estudos de balanço de sedimento (sediment budgets) sejam desenvolvidos concomitantemente ao estudo de contribuição das fontes de sedimento.

\section{CONCLUSÕES}

1. O manejo inadequado do solo nas áreas de lavoura, a falta de planejamento das vias de acesso e a ausência de práticas de controle do escoamento superficial, que sejam compatíveis com a fragilidade condicionada pelos solos e pelo relevo da bacia hidrográfica, têm provocado o surgimento de processos erosivos acelerados com efeitos negativos ao agricultor e à sociedade.
2. As estradas apresentam alta porcentagem de contribuição na transferência de sedimentos, mas a contribuição das áreas de lavoura aumenta em precipitações pluviais de alta magnitude. Isso evidencia que a magnitude dos eventos de chuva influencia a proporção de contribuição entre as fontes ao longo do ano na bacia hidrográfica, interferindo no processo de mobilização de sedimentos e nutrientes em direção à rede de drenagem.

\section{AGRADECIMENTOS}

Ao Conselho Nacional de Desenvolvimento Científico e Tecnológico $(\mathrm{CNPq})$ e à Fundação de Amparo à Pesquisa do Estado do Rio Grande do Sul (FAPERGS), pelo apoio financeiro.

\section{LITERATURA CITADA}

BIRD, G.; BREWER, P.A.; MACKLIN, M.G.; NIKOLOVA, M.; KOTSEV, T.; MOLLOV, M. \& SWAIN, C. Quantifying sediment-associated metal dispersal using $\mathrm{Pb}$ isotopes: Application of binary and multivariate mixing models at the catchment-scale. Environ. Pollut., 158:2158-2169, 2010 .

CAITCHEON, G.G. Applying environmental magnetism to sediment tracing. Tracers Hydrol., 215:285-292, 1993.

CASÃO JUNIOR, R.; ARAÚJO, A.G. \& LLANILLO, R.F. Notill agriculture in southern Brazil. Londrina, IAPAR/FAO, 2012. 77 p.

COLLINS, A.L.; WALLING, D.E. \& LEEKS, G.J.L. Source type ascription for fluvial suspended sediment based on a quantitative composite fingerprinting technique. Catena, 29:1-27, 1997.

COLLINS, A.L. \& WALLING, D.E. Selecting fingerprint properties for discriminating potential suspended sediment sources in river basins. J. Hydrol., 261:218-244, 2002.

COLLINS, A.L. \& WALLING, D.E. Documenting catchment suspended sediment sources: Problems, approaches and prospects. Prog. Phys. Geogr., 28:159-196, 2004.

COLLINS, A.L.; WALLING, D.E.; McMELLIN, G.K.; ZHANG, Y.; GRAY, J.; McGONIGLE, D. \& CHERRINGTON, R. A preliminary investigation of the efficacy of riparian fencing schemes for reducing contributions from eroding channel banks to the siltation of salmonid spawning gravels across the south west UK. J. Environ. Manage., 91:1341-1349, $2010 \mathrm{a}$

COLlins, A.L.; WALLING, D.E.; WEBB, L. \& KING, P. Apportioning catchment scale sediment sources using a modiûed composite ûngerprinting technique incorporating property weightings and prior information. Geoderma, 155:249-261, 2010b. 
COLLINS, A.L.; ZHANG, Y.; WALLING, D.E.; GRENFELL, S.E. \& SMITH, P. Tracing sediment loss from eroding farm tracks using a geochemical ûngerprinting procedure combining local and genetic algorithm optimization. Sci. Total Environ., 408:5461-5471, 2010c.

COLLINS, A.L.; ZHANG, Y.; WALLING, D.E.; GRENFELL, S.E.; SMITH, P.; GRISCHEFF, J.; LOCKE, A.; SWEETAPPLE, A. \& BROGDEN, D. Quantifying finegrained sediment sources in the River Axe catchment, southwest England: application of a Monte Carlo numerical modelling framework incorporating local and genetic algorithm optimisation. Hydrol. Proces., 26:19621983, 2012a.

COLLINS, A.L.; ZHANG, Y.; McCHESNEY, D.; WALLING, D.E.; HALEY, S.M. \& SMITH, P. Sediment source tracing in a lowland agricultural catchment in southern England using a modified procedure combining statistical analysis and numerical modeling. Sci. Total Environ., 414:301317, 2012b.

D'HAEN, K.; Verstraeten, G. \& Degryse, P. Fingerprinting historical fluvial sediment fluxes. Progr. Phys. Geogr., 36:154-186, 2012.

DAY, S.S.; GRAN, K.B.; BELMONT, P. \& WAWRZYNIEC, T. Measuring bluff erosion. Part 2: Pairing aerial photographs and terrestrial laser scanning to create a watershed scale sediment budget. Earth Surf. Proces. Landf., 38:1055-1156, 2013.

DU, J.; WU, Y.; HUANG, D. \& ZHANG, J. Use of ${ }^{7} \mathrm{Be},{ }^{210} \mathrm{~Pb}$ and ${ }^{137} \mathrm{Cs}$ tracers to the transport of surface sediments of the Changjiang Estuary, China. J. Marine Syst., 82:286$294,2010$.

EMPRESA BRASILERIA DE PESQUISA AGROPECUÁRIA EMBRAPA. Centro Nacional de Pesquisa de Solos. Manual de métodos de análise de solo. 2.ed. Rio de Janeiro, 1997. 212p.

ESTRANY, J.; GARCIA, C. \& WALLING, D.E. An investigation of soil erosion and redistribution in a Mediterranean lowland agricultural catchment using caesium-137. Inter. J. Sed. Res., 25:1-16, 2010.

FEDERAÇÃO BRASILEIRA DE PLANTIO DIRETO NA PALHA - FEBRAPDP. Brazilian Federation of Direct Sowing in Straw. 2011. Disponível em: <http:// www.febrapdp.org.br>. Acesso em: 20 maio 2013.

GRANGER, S.J.; BOL, R.; HAWKINS, J.M.B.; WHITE, S.M.; NADEN, P.S.; OLD, G.H.; MARSH, J.K.; BILOTTA, G.S.; BRAZIER, R.E.; MACLEOD, C.J.A. \& HAYGARTH, P.M. Using artificial fluorescent particles as tracers of livestock wastes within an agricultural catchment. Sci. Total Environ., 409:1095-1103, 2011.

INSTITUTO BRASILEIRO DE GEOGRAFIA E ESTATÍSTICA - IBGE. Levantamento de recursos naturais do projeto RadamBrasil. (1986). Folha SH.22. Porto Alegre e parte das folhas SH.21 Uruguaiana e SI.22 Lagoa Mirim. Rio de Janeiro, 1986. 796p.

JOHNSON, R.A. \& WICHERN, D.W. Applied multivariate statistical analysis. London, Prentice-Hall International, 1992. 642p.
KAMINSKI, J.; RHEINHEIMER, D.S.; GATIBONI, L.C.; BRUNETTO, G. \& SILVA, L.S. Eficiência da calagem superficial e incorporada precedendo o sistema plantio direto em um Argissolo sob pastagem natural. R. Bras. Ci. Solo, 29:573-580, 2005

KREIN, A.; PETTICREW, E. \& UDELHOVEN, T. The use of fine sediment fractal dimensions and colour to determine sediment sources in a small watershed. Catena, 53:165179, 2003.

LINDAHL, P.; LEE, S.H.; WORSFOLD, P. \& KEITH-ROACH, M. Plutonium isotopes as tracers for ocean processes: A review. Marine Environ. Res., 69:73-84, 2010.

MARTÍNEZ-CARRERAS, N.; KREIN, A.; UDELHOVEN, T.; GALLART, F.; IFFLY, J.F.; HOFFMANN, L.; PFISTER, L. \& WALLING, D.E. A rapid spectral-reflectance-based fingerprinting approach for documenting suspended sediment sources during storm runoff events. J. Soil Sed., 10:400-413, 2010a.

MARTÍNEZ-CARRERAS, N.; KREIN, A.; GALLART, F.; IFFLY, J.F.; PFISTER, L.; HOFFMANN, L. \& OWENS, P.N. Assessment of different colour parameters for discriminating potential suspended sediment sources and provenance: A multi-scale study in Luxembourg. Geomorphology, 118:118-129, 2010b.

MARTÍNEZ-CARRERAS, N.; UDELHOVEN, T.; KREIN, A.; GALLART, F.; IFFLY, J.F.; ZIEBEL, J.; ZIEBEL, J.; HOFFMANN, L.; PFISTER, L. \& WALLING, D.E. The use of sediment colour measured by diffuse reflectance spectrometry to determine sediment sources: Application to the Attert River catchment (Luxembourg). J. Hydrol., 382:49-63, 2010c.

MEHRA, O.P. \& JACKSON, M.C. Iron oxide removal from soil and clay by dithionite-citrate system buffered with sodium bicarbonate. In: NATIONAL CONFERENCE ON CLAY MINERALS, 7., Washington, 1960. Proceedings... Washington, Pergamon Press, 1960. p.317-327.

MINELLA, J.P.G.; WALLING, D.E. \& MERTEN, G. Combining sediment source tracing techniques with traditional monitoring to assess the impact of improved land management on catchment sediment yields. J. Hydrol., 348:546-563, 2008.

MINELLA, J.P.G. \& MERTEN, G.H. Monitoramento de bacias hidrográficas para identificar fontes de sedimentos em suspensão. Ci. Rural, 41:424-432, 2011.

MOTHA, J.A.; WALLBRINK, P.J.; HAIRSINE, P.B. \& GRAYSON, R.B. Determining the sources of suspended sediment in a forested catchment in Southeastern Australia. Water Res. Resour., 39:1056-1069, 2003.

MURPHY, J. \& RILEY, J.P. A modified single solution method for the determination of phosphate in natural waters. Anal. Chim. Acta., 27:31-36, 1962.

NOSRATI, K.; GOVERS, G.; AHMADI, H.; SHARIFI, F.; AMOOZEGAR, M.A.; MERCKX, R. \& VANMAERCKE, M. An exploratory study on the use of enzyme activities as sediment tracers: Biochemical fingerprints? Inter. J. Sed. Res., 26:136-151, 2011. 
OWENS, P.L. \& WALLING, D.E. Changes in sediment sources and floodplain deposition rates in the catchment of the River Tweed, Scotland, over the last 100 years: The impact of climate and land use change. Earth Surf. Proces. Landf., 27:403-423, 2002.

PHILliPS, J.M.; RUSSEL, M.A. \& WALLING, D.E. Timeintegrated sampling of fuvial suspended sediment: a simple methodology for small catchments. Hydrol. Proces., 14:2589-2602, 2000.

REICHERT, J.M.; SCHÄFER, M.J.; ELTZ, F.L.F. \& NORTON, L.D. Erosão em sulcos e entressulcos em razão do formato de parcela em Argissolo VermelhoAmarelo arênico. R. Bras. Ci. Solo, 36:965-973, 2001.

ROBAINA, L.E.S.; TRENTIN, R.; BAZZAN, T.; RECKZIEGEL, E.W.; VERDUM, R. \& NARDIN, D.D. Compartimentação geomorfológica da bacia hidrográfica do Ibicuí, Rio Grande do Sul, Brasil: proposta de classificação. R. Bras. Geomorf., 11:11-23, 2010.

ROSSATO, M.S. Os climas do Rio Grande do Sul: variabilidade, tendências e tipologia. Porto Alegre, Universidade Federal do Rio Grande do Sul, RS, 2011. 240p. (Tese de Doutorado)

SLATTERY, M.C.; BURT, T.P. \& WALDEN, J. The application of mineral magnetic measurements to quantify within-storm variations in suspended sediment source. Inter. Assoc. Hydrol. Sci. Public., 229:143-151, 1995.

SMITH, J.P.; BULLEN, T.D.; BRABANDER, D.J. \& OLSEN, C.R. Strontium isotope record of seasonal scale variations in sediment sources and accumulation in low-energy, subtidal areas of the lower Hudson River estuary. Chem. Geol., 264:375-384, 2009.
SOUZA, E.D.; COSTA, S.E.V.G.A.; ANGHINONI, I.; CARVALHO, P.C.F.; OLIVEIRA, E.V.F.; MARTINS, A.P.; CAO, E. \& ANDRIGHETTI, M. Soil aggregation in a crop-livestock integration system under no-tillage. R. Bras. Ci. Solo, 34:1365-1374, 2010.

STRAHLER, A.N. Quantitative analysis of watershed geomorphology. Trans. Am. Geophys. Union, 38:913-920, 1957.

TIECHER, T.; RHEINHEIMER, D.S. \& CALEGARI, A. Soil organic phosphorus forms under different soil management systems and winter crops, in a long term experiment. Soil Till. Res., 124:57-67, 2012.

WALLING, D.E. Tracing suspended sediment sources in catchments and river systems. Sci. Total Environ., 344:159-184, 2005.

WALLING, D.E. \& WOODWARD, J.C. Tracing sources of suspended sediment in river basins: A case study of the River Culm, Devon, UK. Marine Freshwat. Res., 46:327336, 1995.

WALLING, D.E. \& COLLINS, A.L. Integrated assessment of catchment sediment budgets: A technical manual. Exeter, Department for International Development, 2000. 168p.

WALlinG, D.E.; COLLINS, A.L. \& STROUD, R.W. Tracing suspended sediment and particulate phosphorus sources in catchments. J. Hydrol., 350:74-289, 2008.

WU, W.; XU, S.; LU, H.; YANG, J.; YIN, H. \& LIU, W. Mineralogy, major and trace element geochemistry of riverbed sediments in the headwaters of the Yangtze, Tongtian River and Jinsha River. J. Asian Earth Sci., 40:611-621, 2011.

YU, L. \& OLDFIELD, F. A multivariate mixing model for identifying sediment source from magnetic measurements. Quater. Res., 32:168-181, 1989. 